Mathematical Modelling and Analysis

Volume 20 Number 1, January 2015, 94-111

http://dx.doi.org/10.3846/13926292.2015.1003108

(c) Vilnius Gediminas Technical University, 2015
Publisher: Taylor\&Francis and VGTU

http://www.tandfonline.com/TMMA

ISSN: $1392-6292$

eISSN: $1648-3510$

\title{
A Priori Estimation of a Time Step for Numerically Solving Parabolic Problems*
}

\author{
Petr N. Vabishchevich ${ }^{a, b}$ \\ ${ }^{a}$ Nuclear Safety Institute, Russian Academy of Sciences \\ 52, B. Tulskaya, 115191 Moscow, Russia \\ ${ }^{b}$ North-Eastern Federal University \\ 58, Belinskogo, 677000 Yakutsk, Russia \\ E-mail: vabishchevich@gmail.com
}

Received May 7, 2014; revised December 10, 2014; published online January 15, 2015

\begin{abstract}
This work deals with the problem of choosing a time step for the numerical solution of boundary value problems for parabolic equations. The problem solution is derived using the fully implicit scheme, whereas a time step is selected via explicit calculations. The selection strategy consists of the following two stages. At the first stage, we employ explicit calculations for selecting the appropriate time step. At the second stage, using the implicit scheme, we calculate the solution at a new time level. This solution should be close to the solution of our problem at this time level with a prescribed accuracy. Such an algorithm leads to explicit formulas for the calculation of the time step and takes into account both the dynamics of the problem solution and changes in coefficients of the equation and in its right-hand side. The same formulas for the evaluation of the time step are obtained by using a comparison of two approximate solutions, which are obtained using the explicit scheme with the primary time step and the step that is reduced by half. Numerical results are presented for a model parabolic boundary value problem, which demonstrate the robustness of the developed algorithm for the time step selection.
\end{abstract}

Keywords: parabolic equation, finite difference schemes, explicit schemes, implicit schemes, time step.

AMS Subject Classification: 65J08; 65M06; 65M12.

\section{Introduction}

In numerically solving boundary value problems for time-dependent equations, emphasis is on discretizations in time $[1,2,7]$. For parabolic equations of second order, unconditionally stable schemes are constructed using implicit approximations $[9,10,11]$. Two-level schemes are commonly used in computational practice, whereas multilevel schemes occur more rarely. For unconditionally

* This work was supported by RFBR (project 14-01-00785). 
stable schemes, a time step is selected only due to the accuracy of the approximate solution.

The problem of how to control the time step is relatively well resolved for the numerical solution of the Cauchy problem for systems of differential equations $[3,5,6]$. The basic approach involves the following stages. First, we perform additional calculations in order to estimate the error of the approximate solution at a new time level. Further, a time step is estimated using the theoretical asymptotic dependence of accuracy on a time step. After that we decide whether it is necessary to correct the time step and to repeat calculations.

Additional calculations for estimating the error of the approximate solution may be performed in a different way. In particular, it is possible to obtain an approximate solution using two different schemes that have the same theoretical order of accuracy. The most famous example of this strategy involves the solution of the problem on a separate time interval using a preliminary step (the first solution) and the step reduced by half (the second solution). In numerically solving the Cauchy problem for systems of ordinary differential equations, there are are also applied nested methods, where two approximate solutions of different orders of accuracy are compared.

In the above-mentioned methods of selecting a time step, a posteriori estimation of accuracy is employed. In this case, we decide whether or not this time step is acceptable. If so, we must determine by how much we should increase the time step for future calculations, or if not, by how much we should reduce the time step and re-calculate the solution. Such strategies can be also applied to the approximate solution of unsteady boundary value problems using a more advanced a posteriori analysis $[4,8,13]$.

The conventional technology to select the time step is based on a prediction of the time step using an error analysis under the assumption that the error does not vary essentially in time. Further the predicted time step is applied for performing calculations. At the final stage, analysing the solution obtained at the new time level, we make the decision whether the selected step and the solution at the new time level itself are acceptable, or the step is too large and the solution is not appropriate. In the latter case, we take a smaller time step and calculations are repeated. In predicting the time step, the procedure is of a priori nature, whereas at the stage of obtaining the solution and selecting the time step procedure, we make a posteriori decision, i.e., we conduct calculations with the predicted step and then either accept it or choose a new time step with the following repetition of calculations.

In our work, we propose a new technology how to select the time step, which focuses on the approximate solution of boundary value problems for parabolic equations. For such problems, we apply the implicit approximation in time of first (or rarely second) order. Explicit schemes have too strong restrictions for stability on the allowable time step and so they may be too costly for practical calculations. It should be noted that computational costs for the implicit scheme are usually significantly higher than computational costs for the explicit scheme. We use the specified predicted time step, and control changes of problem parameters (coefficients of the equation, the righthand side). To obtain the solution at a new time level, the backward Euler 
scheme is employed. The time step at the new time level is explicitly calculated (not the solution itself) using two previous time levels and takes into account changes in the equation coefficients and its right-hand side. The time step at the new time level is obtain analytically via explicit formulas. We proposed and demonstrated some possibilities of the new algorithm, which defines an heuristic for selection of adaptive time integration steps. The author does not attempt to analyse the optimality (efficiency) of this new strategy.

The paper is organized as follows. In Section 2, we consider a Cauchy problem for a system of linear ordinary differential equations that is obtained from numerically solving boundary value problems for parabolic equations after discretization in space. For the approximate solution, estimates for stability are presented along with estimates for accuracy in the corresponding Hilbert space. Formulas for the selection of a time step are obtained in Section 3 using a comparison of the problem solutions corresponding to the forward time level and backward one. In Section 4, we show that similar expressions for a time step can be obtained via making a comparison of the solutions derived with one time step and two half steps. Section 5 presents numerical results for a model boundary value problem for a one-dimensional parabolic equation obtained on the basis of the developed algorithm for selecting a time step. In Section 6, we discuss the more general problems.

\section{Model Problem}

Let us consider the Cauchy problem for the linear equation

$$
\frac{d u}{d t}+A(t) u=f(t), \quad 0<t \leq T
$$

supplemented with the initial condition

$$
u(0)=u_{0}
$$

The problem is investigated in a finite-dimensional Hilbert space $H$. Assume that

$$
A(t) \geq 0
$$

in $H$. Due to the non-negativity of the operator $A$, for the problem (2.1), (2.2), we have the following estimate for stability with respect for the initial data and the right-hand side:

$$
\|u(t)\| \leq\left\|u_{0}\right\|+\int_{0}^{t}\|f(\theta)\| d \theta .
$$

The problem (2.1), (2.2) results from finite difference, finite volume or finite element approximations (lumped masses scheme [12]) for numerically solving boundary value problems for a parabolic equation of second order. In this problem, an unknown function $u(\boldsymbol{x}, t)$ satisfies the equation

$$
\frac{\partial u}{\partial t}-\sum_{\alpha=1}^{m} \frac{\partial}{\partial x_{\alpha}}\left(k(\boldsymbol{x}, t) \frac{\partial u}{\partial x_{\alpha}}\right)+c(\boldsymbol{x}, t) u=f(\boldsymbol{x}, t), \quad \boldsymbol{x} \in \Omega, 0<t \leq T,
$$


where $\underline{k} \leq k(\boldsymbol{x}) \leq \bar{k}, \boldsymbol{x} \in \Omega, \underline{k}>0, c(\boldsymbol{x}, t) \geq 0$. The equation is complemented by the Dirichlet boundary conditions

$$
u(\boldsymbol{x}, t)=g(\boldsymbol{x}, t), \quad \boldsymbol{x} \in \partial \Omega, 0<t \leq T,
$$

and the initial condition

$$
u(\boldsymbol{x}, 0)=u_{0}(\boldsymbol{x}), \quad \boldsymbol{x} \in \Omega .
$$

To solve numerically this time-dependent problem, we introduce a nonuniform grid in time:

$$
t_{0}=0, \quad t_{n+1}=t_{n}+\tau_{n+1}, \quad n=0,1, \ldots, N-1, \quad t_{N}=T .
$$

We will employ notation $f_{n}=f\left(t_{n}\right)$. For the problem (2.1), (2.2), we apply the fully implicit scheme, where the transition from the current time level to the next one is performed as follows:

$$
\frac{y_{n+1}-y_{n}}{\tau_{n+1}}+A_{n+1} y_{n+1}=f_{n+1}, \quad n=0,1, \ldots, N-1
$$

starting from the initial condition

$$
y_{0}=u_{0}
$$

Under the restriction $A_{n+1} \geq 0$, from (2.4), it follows immediately that the approximate solution satisfies the level-wise estimate

$$
\left\|y_{n+1}\right\| \leq\left\|y_{n}\right\|+\tau_{n+1}\left\|f_{n+1}\right\|
$$

Thus, we obtain the discrete analog of the estimate (2.3):

$$
\left\|y_{n+1}\right\| \leq\left\|u_{0}\right\|+\sum_{k=0}^{n} \tau_{k+1}\left\|f_{k+1}\right\|
$$

corresponding to the problem (2.4), (2.5). For the error $z_{n}=y_{n}-u_{n}$ of the approximate solution, we have the problem

$$
\frac{z_{n+1}-z_{n}}{\tau_{n+1}}+A_{n+1} z_{n+1}=\psi_{n+1}, \quad n=0,1, \ldots, N-1, \quad z_{0}=0
$$

Here $\psi_{n+1}$ stands for the truncation error:

$$
\psi_{n+1}=f_{n+1}-\frac{u_{n+1}-u_{n}}{\tau_{n+1}}+A_{n+1} u_{n+1} .
$$

Similarly to (2.6), we get the estimate for the error:

$$
\left\|z_{n+1}\right\| \leq \sum_{k=0}^{n} \tau_{k+1}\left\|\psi_{k+1}\right\| .
$$


If $\left\|\psi_{k+1}\right\| \leq \delta$, then for the error, we have

$$
\left\|z_{n+1}\right\| \leq \delta t_{n+1} .
$$

Therefore, to control the error, we can employ the summarized error $\tau_{n+1} \delta$ over the interval $t_{n} \leq t \leq t_{n+1}$. In this case, a value $\delta$ defines the same level of the error over the entire interval of integration.

The main feature of our approach is that in numerical solving time-dependent problems the computations are done by using the stable implicit scheme. The explicit schemes are used only virtually (theoretically) in order to estimate residual of the implicit scheme and therefore the stability is fully defined by the properties of the basic implicit scheme.

It is clear that the proposed strategy for choosing the time step is heuristic. It is based on the assumption that the approximate solution of the unsteady problem, obtained using the implicit scheme, at the transition the the new time level is close in sense of accuracy to the approximate solution of the same problem, obtained using the explicit scheme with the same approximation order as the implicit scheme.

The proposed global strategy of using low-cost explicit schemes to estimate the time step for implicit schemes in solving IVBP for parabolic equations is illustrated by the example with the explicit and implicit Euler schemes, which are of the first-order convergence in time. We also provide an example of using explicit approximations of the second-order convergence for the evaluation of the time step in the solution of parabolic problems using the Crank-Nicolson scheme.

\section{Algorithm for Estimation of a Time Step}

In view of the estimates for the error of the approximate solution (2.8), the accumulation of the error during the transition from the time level $t_{n}$ to a new temporary level $t_{n+1}$ is governed by the rule

$$
\left\|z_{n+1}\right\| \leq\left\|z_{n}\right\|+\tau_{n+1}\left\|\psi_{n+1}\right\| .
$$

Because of this, we have to control the local error $\psi_{n+1}$.

If we can calculate the truncation error $\psi_{n+1}$, then it will be possible to get an a posteriori estimate of the error. Comparing $\left\|\psi_{n+1}\right\|$ with the prescribed error level $\delta$, this makes possible to evaluate the quality of the choice of the time step $\tau_{n+1}$. Namely, if $\left\|\psi_{n+1}\right\|$ is much larger (smaller) than $\delta$, then the time step is taken too large (small), and if $\left\|\psi_{n+1}\right\|$ is close to $\delta$, then this time step is optimal. Thus, we want

$$
\tau_{n+1}:\left\|\psi_{n+1}\right\| \approx \delta .
$$

The problem is that we cannot evaluate the truncation error, since it is determined using the exact solution that is unknown. Because of this, we must focus on some estimates for the truncation error that guarantee the fulfilment of $(3.1)$.

The general approach to the adaptive choice of the time step for solving unsteady problems includes the following key elements: 
- performing a selection of the predicted time step via an analysis of the solution from the previous time levels;

- conducting calculations with the predicted time step;

- conducting an analysis for the accuracy of the obtained approximate solution and carrying out the recalculation with a smaller time step if necessary.

This general strategy is usually implemented (see, e.g, $[3,5,6])$ using the asymptotic analysis for the error of the approximate solution under the assumption that the error does not vary essentially in time. The main features of our approach to selecting the time step are presented below.

In our case, the predicted time step is fixed. To estimate the step at the new time level (during the transition from the time level $t_{n}$ to the next one $t_{n+1}$ ), we focus on the previous time step $\tau_{n}=t_{n}-t_{n-1}$. First of all, we are interested in the possibility to employ a larger time step at the new time level. In view of this, the predicted time step is defined as follows:

$$
\widetilde{\tau}_{n+1}=\gamma \tau_{n}
$$

where $\gamma$ is a numerical parameter. The factor $\gamma$ for the maximum increase of the time step is defined, for example, to be equal to 1.25 or 1.5 . The problem parameters (the coefficients of the equation and the right-hand side) are estimated over the interval $\left[t_{n}, t_{n}+\widetilde{\tau}_{n+1}\right]$. In estimating the time step, we should not miss the time moment, where essential changes in the parameters of the problem are observed.

The choice of the time step under the restriction $\tau_{n+1} \leq \widetilde{\tau}_{n+1}$ is performed using calculation formulas based on the implicit error estimate at the new time level. The approximate solution at the new time level is evaluated by the implicit scheme (2.4), whereas estimating the time step is carried out via the explicit scheme. Both the implicit and explicit schemes have the same order of approximation and they are considered with the same initial conditions (at $t=t_{n}$ ). We perform a small number (one or two) of time steps, and therefore, possible computational instability for the explicit scheme has no time to appear. Because of this, we can expect that such approximate solutions are close to each other. On the basis of this closeness, we evaluate the error of the approximate solution and obtain the calculation formula for the time step.

Among possible variants for the correction of the time step, we consider the following technique. The step $\tau_{n+1}$ is selected from the conditions:

Forward step. Using the explicit scheme, we find the auxiliary solution $v_{n+1}$ at the time level $t_{n+1}$;

Backward step. From the obtained $v_{n+1}$, applying backward in time consideration, we determine $v_{n}$ at the time level $t_{n}$ via explicit formulas;

Step selecting. The step $\tau_{n+1}$ is evaluated via closeness between $v_{n}$ and $y_{n}$.

In fact, we carry out the back analysis of the error of the approximate solution over the interval $t_{n} \leq t \leq t_{n+1}$ using two schemes (explicit and implicit) of the same accuracy. 
Let us present the formulas for selecting a time step. The solution $v_{n+1}$ is determined from the equation

$$
\frac{v_{n+1}-y_{n}}{\tau_{n+1}}+A_{n} y_{n}=f_{n}
$$

For $v_{n}$, we have

$$
\frac{v_{n+1}-v_{n}}{\tau_{n+1}}+A_{n+1} v_{n+1}=f_{n+1} .
$$

From (3.2), (3.3), we immediately get

$$
v_{n}-y_{n}=\tau_{n+1}\left(A_{n+1}-A_{n}\right) y_{n}-\tau_{n+1}\left(f_{n+1}-f_{n}\right)+\tau_{n+1}^{2} A_{n+1}\left(f_{n}-A_{n} y_{n}\right) .
$$

The first two terms are associated with the time derivative applied to the problem operator and to the right-hand side. To evaluate them approximately, it seems reasonable to use the time step from the previous time level. But this may be inconvenient to implement. For instance, we have

$$
\tau_{n+1}\left(f_{n+1}-f_{n}\right)=\tau_{n+1}^{2} \frac{f_{n+1}-f_{n}}{\tau_{n+1}},
$$

and therefore we have to evaluate the difference derivative of the right-hand side for $t_{n} \leq t \leq t_{n+1}$. The problem is that the derivation of such estimates involves the unknown value $t_{n+1}$. The simplest approach is to evaluate this derivative using the previous time step:

$$
\frac{f_{n+1}-f_{n}}{\tau_{n+1}} \approx \frac{f\left(t_{n}+\tau_{n}\right)-f_{n}}{\tau_{n}} .
$$

But in this case, if $\tau_{n+1}>\tau_{n}$, then we cannot detect significant changes in the right-hand side for $t_{n}+\tau_{n} \leq t \leq t_{n}+\tau_{n+1}$.

To resolve the problem, it is possible to use the standard methods available to control a time step for numerically solving time-dependent problems. The first method restricts the growth of the time step with respect to the previous value. We set

$$
\tau_{n+1} \leq \gamma \tau_{n}
$$

where $\gamma>1$, introduced above, is a numerical parameter controlling the increase of the time step. The second requirement is that the step cannot be too small:

$$
\tau_{n+1} \geq \tau_{0}
$$

where $\tau_{0}$ is a specified minimum time step.

Under the assumption (3.5), we can estimate the time derivative of the right-hand side, putting

$$
\frac{f_{n+1}-f_{n}}{\tau_{n+1}} \approx \frac{f\left(t_{n}+\gamma \tau_{n}\right)-f_{n}}{\gamma \tau_{n}} .
$$

Therefore

$$
\begin{aligned}
& \tau_{n+1}\left(f_{n+1}-f_{n}\right) \approx \frac{\tau_{n+1}^{2}}{\gamma \tau_{n}}\left(\widetilde{f}_{n+1}-f_{n}\right), \\
& \tau_{n+1}\left(A_{n+1}-A_{n}\right) y_{n} \approx \frac{\tau_{n+1}^{2}}{\gamma \tau_{n}}\left(\widetilde{A}_{n+1}-A_{n}\right) y_{n},
\end{aligned}
$$


where

$$
\tilde{f}_{n+1}=f\left(t_{n}+\gamma \tau_{n}\right), \quad \widetilde{A}_{n+1}=A\left(t_{n}+\gamma \tau_{n}\right) .
$$

For the last term in the right-hand side of (3.4), in view of (2.4), we have

$$
\tau_{n+1}^{2} A_{n+1}\left(f_{n}-A_{n} y_{n}\right)=\frac{\tau_{n+1}^{2}}{\tau_{n}} A_{n+1}\left(y_{n}-y_{n-1}\right) .
$$

With accuracy up to $O\left(\tau_{n+1}^{3}\right)$, we put

$$
\tau_{n+1}^{2} A_{n+1}\left(f_{n}-A_{n} y_{n}\right) \approx \frac{\tau_{n+1}^{2}}{\tau_{n}} \widetilde{A}_{n+1}\left(y_{n}-y_{n-1}\right) .
$$

With this in mind, the equality (3.4) is replaced by the approximate equality:

$$
v_{n}-y_{n} \approx \frac{\tau_{n+1}^{2}}{\tau_{n}}\left(\frac{1}{\gamma}\left(\widetilde{A}_{n+1}-A_{n}\right) y_{n}-\frac{1}{\gamma}\left(\widetilde{f}_{n+1}-f_{n}\right)+\widetilde{A}_{n+1}\left(y_{n}-y_{n-1}\right)\right) .
$$

The value of $v_{n}-y_{n}$ we associate with the solution error over the interval $t_{n} \leq t \leq t_{n+1}$. Because of this, we set

$$
\left\|v_{n}-y_{n}\right\| \leq \tau_{n+1} \delta
$$

From (3.8), we have

$$
\left.\left\|v_{n}-y_{n}\right\| \lesssim \frac{\tau_{n+1}^{2}}{\tau_{n}}\left(\frac{1}{\gamma} \| \widetilde{A}_{n+1}-A_{n}\right) y_{n}\left\|+\frac{1}{\gamma}\right\| \widetilde{f}_{n+1}-f_{n}\|+\| \widetilde{A}_{n+1}\left(y_{n}-y_{n-1}\right) \|\right) .
$$

In view of (3.5), (3.6), (3.9), from (3.8), we obtain the following formula for calculating the time step:

$$
\begin{aligned}
\tau_{n+1} & =\max \left\{\tau_{0}, \min \left\{\gamma, \gamma_{n+1}\right\} \tau_{n}\right\} \\
\gamma_{n+1} & =\delta\left(\frac{1}{\gamma}\left\|\left(\widetilde{A}_{n+1}-A_{n}\right) y_{n}\right\|+\frac{1}{\gamma}\left\|\tilde{f}_{n+1}-f_{n}\right\|+\left\|\widetilde{A}_{n+1}\left(y_{n}-y_{n-1}\right)\right\|\right)^{-1} .
\end{aligned}
$$

This formula for selecting a time step reflects clearly (see the denominator in the expression for $\gamma_{n+1}$ ) corrective actions, which are related to the timedependence of the problem operator (the first part) and the right-hand side (the second part) as well as to the time-variation of the solution itself (the third part).

The above considerations are given for the derivation of formulas (3.10) for the time step at the new time level. No direct calculations of auxiliary variables $v_{n+1}$ and $v_{n}$ by explicit schemes (3.2), (3.3) are conducted. To employ a priori estimate (3.10) for the time step, we involve information on the known solution at two previous time levels $y_{n}$ and $y_{n+1}$ as well as the coefficients and the right-hand side at time $t_{n}$ and at the next time moment $t_{n}+\gamma \tau_{n}$. The computational complexity of the algorithm for determining the time step (3.10) can be easy estimated; for typical problems, it is small in comparison with the implementation of the fully implicit scheme. The step is estimated through the use of explicit schemes. Their instability is practically insignificant within two steps. 


\section{Estimation of a Time Step on the Basis of Step Dou- bling}

Above we have considered the procedure for the error estimation based on the numerical solution of two subproblems over the intervals $\left[t_{n}, t_{n+1}\right]$ and $\left[t_{n+1}, t_{n}\right]$ using explicit schemes. To solve numerically the Cauchy problem, the traditional strategy is to select an integration step using a comparison of the approximate solution obtained by the preliminary step with the solution calculated with the step reduced by half. A similar idea for evaluating truncation error using the approximate solutions on the intervals $\left[t_{n}, t_{n+1}\right]$ and $\left[t_{n}, t_{n+1 / 2}\right]$, $\left[t_{n+1 / 2}, t_{n+1}\right]$, we can apply to our approach for controlling the time step.

For numerically solving problem (2.1), (2.2), we use fully implicit scheme (2.4), (2.5). We employ the explicit scheme over the interval $t_{n} \leq t \leq t_{n+1}$ in order to select the time step $\tau_{n+1}$. The selection strategy includes:

Calculation with an integer step. Using the explicit scheme, we determine the solution $v_{n+1}$ at the time level $t_{n+1}$ via the step $\tau_{n+1}$;

Calculation with a half-integer step. Using the explicit scheme, we calculate the solution $w_{n+1}$ at the time level $t_{n+1}$ employing the step $0.5 \tau_{n+1}$;

Step selecting. The step $\tau_{n+1}$ is evaluated through the closeness between $v_{n+1}$ and $w_{n+1}$.

For $v_{n+1}$, we have $(3.2)$, and $w_{n+1}$ is determined as follows:

$$
\begin{aligned}
& \frac{w_{n+1 / 2}-y_{n}}{0.5 \tau_{n+1}}+A_{n} y_{n}=f_{n}, \\
& \frac{w_{n+1}-w_{n+1 / 2}}{0.5 \tau_{n+1}}+A_{n+1 / 2} w_{n+1 / 2}=f_{n+1 / 2} .
\end{aligned}
$$

Eliminating $w_{n+1 / 2}$ from (4.1), (4.2), we get

$$
\begin{aligned}
w_{n+1}= & y_{n}-\frac{\tau_{n+1}}{2}\left(A_{n+1 / 2}+A_{n}\right) y_{n} \\
& +\frac{\tau_{n+1}}{2}\left(f_{n+1 / 2}+f_{n}\right)-\frac{\tau_{n+1}^{2}}{4} A_{n+1 / 2}\left(f_{n}-A_{n} y_{n}\right) .
\end{aligned}
$$

Because of this, we have

$$
\begin{aligned}
v_{n+1}-w_{n+1}= & \frac{\tau_{n+1}}{2}\left(A_{n+1 / 2}-A_{n}\right) y_{n} \\
& -\frac{\tau_{n+1}}{2}\left(f_{n+1 / 2}-f_{n}\right)+\frac{\tau_{n+1}^{2}}{4} A_{n+1 / 2}\left(f_{n}-A_{n} y_{n}\right) .
\end{aligned}
$$

In view of the above notation (3.5), we employ the approximate expressions:

$$
\begin{aligned}
\frac{\tau_{n+1}}{2}\left(f_{n+1 / 2}-f_{n}\right) & \approx \frac{\tau_{n+1}^{2}}{4 \gamma \tau_{n}}\left(\widetilde{f}_{n+1}-f_{n}\right), \\
\frac{\tau_{n+1}}{2}\left(A_{n+1 / 2}-A_{n}\right) y_{n} & \approx \frac{\tau_{n+1}^{2}}{4 \gamma \tau_{n}}\left(\widetilde{A}_{n+1}-A_{n}\right) y_{n} .
\end{aligned}
$$


By (2.4), we have

$$
\frac{\tau_{n+1}^{2}}{4} A_{n+1 / 2}\left(f_{n}-A_{n} y_{n}\right) \approx \frac{\tau_{n+1}^{2}}{4 \tau_{n}} \widetilde{A}_{n+1}\left(y_{n}-y_{n-1}\right) .
$$

Thus, we arrive at

$v_{n+1}-w_{n+1} \approx \frac{\tau_{n+1}^{2}}{4 \tau_{n}}\left(\frac{1}{\gamma}\left(\widetilde{A}_{n+1}-A_{n}\right) y_{n}-\frac{1}{\gamma}\left(\tilde{f}_{n+1}-f_{n}\right)+\widetilde{A}_{n+1}\left(y_{n}-y_{n-1}\right)\right)$.

The right-hand side of it coincides with the right-hand side of (3.8) with an accuracy of a factor of 4 . Similarly to (3.10), we can formulate the rule for selecting the time step:

$$
\tau_{n+1}=\max \left\{\tau_{0}, \min \left\{\gamma, \widetilde{\gamma}_{n+1}\right\} \tau_{n}\right\}, \quad \widetilde{\gamma}_{n+1}=4 \gamma_{n+1} .
$$

In fact, we have come to the same rule for the estimation of the time step the factor 4 has not any essential matter.

Note that for the estimation of the time step on the basis of step doubling, we solve three auxiliary problems, i.e., over three time intervals $\left[t_{n}, t_{n+1}\right]$, $\left[t_{n}, t_{n+1 / 2}\right],\left[t_{n+1 / 2}, t_{n+1}\right]$. If we use the forward-backward estimation, then we solve only two auxiliary problems, i.e., over two time intervals $\left[t_{n}, t_{n+1}\right]$ and $\left[t_{n+1}, t_{n}\right]$. In view of this, the forward-backward estimation of a time step seems to be more preferable.

\section{$5 \quad$ Numerical Experiments}

The following methodological calculations demonstrate automatic adaptation of the computational algorithm to the basic features of the parabolic problems. It's only a small part an overall review of the problems of the adaptive selection of the time step, but it is absolutely necessary preliminary study. The investigation of the necessity to use variable time steps, the effect of the adaptation the time steps on the accuracy of the approximate solution, computational efficiency of various strategies of choice of the time step in the solution of time-dependent problems requires a separate consideration.

To demonstrate the performance of the proposed algorithm (3.5), (3.9) for selecting a time step based on the implicit scheme for solving the problem (2.1), (2.2), let us consider the boundary value problem for a one-dimensional parabolic equation. Let $u(x, t)$ satisfies the equation

$$
\frac{\partial u}{\partial t}-\frac{\partial^{2} u}{\partial x^{2}}+p(t) u=f(t), \quad 0<x<1,0<t \leq T
$$

as well as the boundary and the initial conditions:

$$
\begin{aligned}
& u(0, t)=0, \quad u(1, t)=0, \quad 0<t \leq T, \\
& u(x, 0)=u_{0}(x), \quad 0<x<1 .
\end{aligned}
$$

To solve approximately the problem (5.1)-(5.3), we apply finite difference discretization in space. Let us introduce a uniform grid with a step $h$ :

$$
\bar{\omega}=\{x \mid x=i h, i=0,1, \ldots, M, M h=1\},
$$


and $\omega$ is the set of interior grid points, whereas $\partial \omega$ is the set of boundary points $(\bar{\omega}=\omega \cup \partial \omega)$. On the set of grid functions such that $u(x)=0, x \notin \omega$, we introduce a Hilbert space $H$, where the inner product and the norm are defined as:

$$
(u, v)=\sum_{x \in \omega} u(x) v(x) h, \quad\|u\|=(u, u)^{1 / 2} .
$$

The grid operator $A(t)$ is written as follows:

$$
A u=-\frac{1}{h^{2}}(u(x+h)-2 u(x)+u(x-h))+p(t) u(x), \quad x \in \omega,
$$

on the set of functions $u(x)=0, x \in \partial \omega$. The operator $A(t)$ is self-adjoint, and if $p(t) \geq 0$, then it is positive definite in $H[9,10,11]$. Thus, after the spatial discretization of (5.1)-(5.3), we arrive to the problem $(2.1),(2.2)$. The Dirichlet boundary conditions are simply injected into this grid operator during the calculations.

As a test problem, we consider the problem (5.1)-(5.3) with $T=0.1$ and the discontinuous coefficient $p(t)$ and the discontinuous $f(t)$ defined as follows:

$$
p(t)=\left\{\begin{array}{ll}
100 t, & 0<t \leq 0.075, \\
0, & 0.075<t \leq 0.1,
\end{array} \quad f(t)= \begin{cases}0, & 0<t \leq 0.05 \\
10 e^{-(t-0.05)}, & 0.05<t \leq 0.1\end{cases}\right.
$$

The problem is solved on the grid with $M=100$, the calculations are performed using the sufficiently small time step $\tau_{1}=\tau_{0}=1 \cdot 10^{-6}$.

First, we consider the case, where the initial condition (5.3) is taken in the following form:

$$
u_{0}(x)=\sin (\pi x), \quad 0<x<1 .
$$

If we specify the error level $\delta=0.1$ and the parameter $\gamma=1.5$, then the timehistory of the time step produced by the algorithm (3.7), (3.10) has the form depicted in Fig. 1. The total number of time steps is $N=268$.

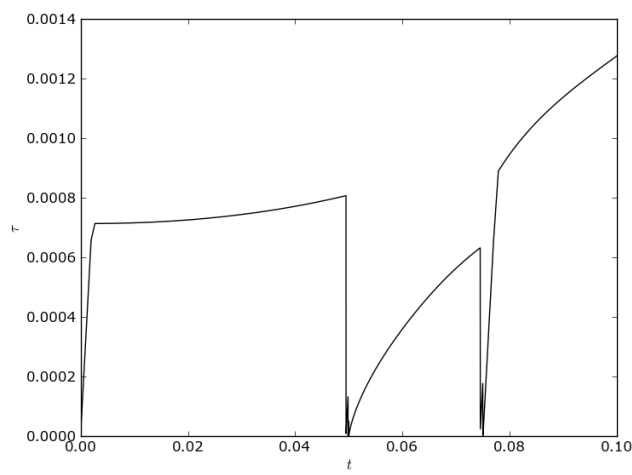

Figure 1. The time-history of the time step: $\delta=0.1, N=268$.

In this figure, we observe essential changes in the value the time step at $t=$ 0.05 and $t=0.075$, i.e., at the time moments corresponding to discontinuities in 
the right-hand side and the coefficient of the equation. In accordance with the rule (3.6), the time step increases at the initial time stage. Let us decompose the correcting coefficient $\gamma_{n+1}$ into three terms:

$$
s_{1}=\frac{1}{\gamma}\left\|\left(\widetilde{A}_{n+1}-A_{n}\right) y_{n}\right\|, \quad s_{2}=\frac{1}{\gamma}\left\|\tilde{f}_{n+1}-f_{n}\right\|, \quad s_{3}=\left\|\widetilde{A}_{n+1}\left(y_{n}-y_{n-1}\right)\right\| .
$$

Figure 2 demonstrates their influence.

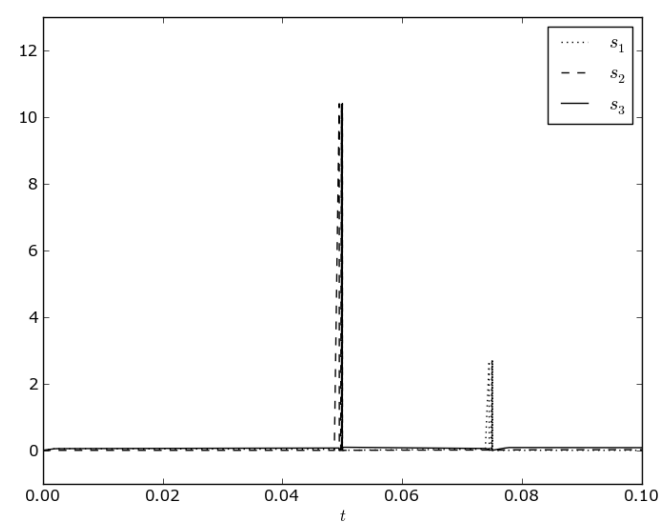

Figure 2. Terms of the correcting coefficient.

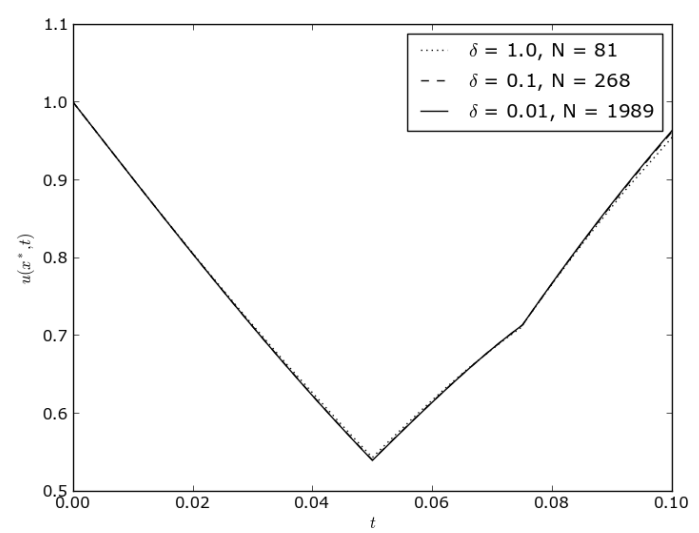

Figure 3. Approximate solution for various $\delta$.

The influence of the reducing error level $\delta$ on the convergence of the approximate solution is shown in Fig. 3. The approximate solution at the point $x^{*}=0.5$ is depicted in this figure. For comparison, Fig. 4 presents similar data that were obtained using the uniform grids in time. 
The number of time steps for the proposed strategy depends not only on the magnitude of the error $\delta$, as it was demonstrated by the data in Fig. 3 . The time step (see (3.10)) is also chosen depending on the minimal step tau as well as on the step increase parameter at the new time level $\gamma$.

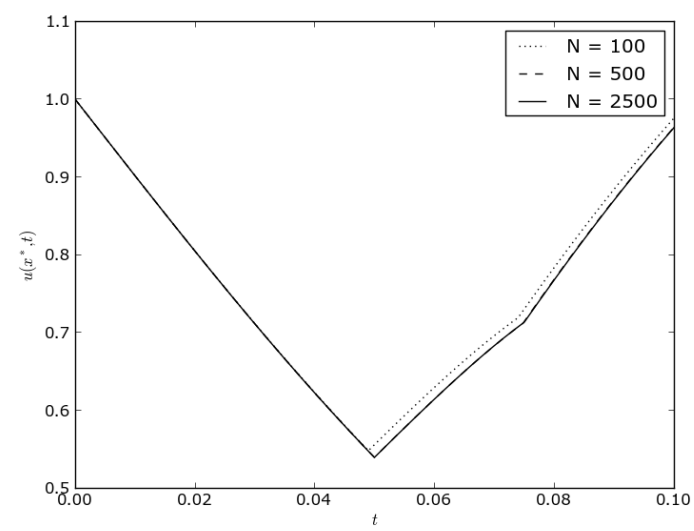

Figure 4. Approximate solution for various uniform time grids.

In Table 1, there are collected data for the number of time steps at different levels of error: $\delta=2^{-\nu}, \nu=0,1, \ldots, 8$. It is easy to observe the theoretically predicted linear dependence of the number of steps on the level of error. Dependence on the minimal step and the increase parameter seems to be rather weak.

Table 1. The number of time steps $N=N\left(\delta, \tau_{0}, \gamma\right)$.

\begin{tabular}{lrrrrrrrrr}
\hline$\nu$ & 0 & 1 & 2 & 3 & 4 & 5 & 6 & 7 & 8 \\
\hline$\tau_{0}=10^{-6}, \gamma=1.25$ & 109 & 151 & 173 & 250 & 419 & 727 & 1328 & 2523 & 4844 \\
$\tau_{0}=10^{-6}, \gamma=1.5$ & 81 & 89 & 165 & 233 & 377 & 698 & 1313 & 2503 & 4836 \\
$\tau_{0}=10^{-6}, \gamma=2$ & 61 & 114 & 145 & 182 & 365 & 689 & 1294 & 2504 & 4819 \\
$\tau_{0}=10^{-7}, \gamma=1.5$ & 83 & 142 & 171 & 239 & 419 & 721 & 1367 & 2595 & 5105 \\
$\tau_{0}=10^{-5}, \gamma=1.5$ & 65 & 92 & 137 & 208 & 345 & 622 & 1163 & 2168 & 3981 \\
\hline
\end{tabular}

Special attention should be given to the influence of the initial conditions. A typical situation is the presence of a boundary layer and this requires to use small steps at the initial time stage. For example, the behaviour of the time step for our model problem with initial conditions

$$
u_{0}(x)= \begin{cases}2 x, & 0<x \leq 0.5 \\ 1-2(x-0.5), & 0.5<x<1\end{cases}
$$

is shown in Fig. 5. Compared with Fig. 1 (smooth initial conditions), the initial time stage is calculated with essentially smaller time steps and the total number of steps is increased by more than a factor of 2 . 


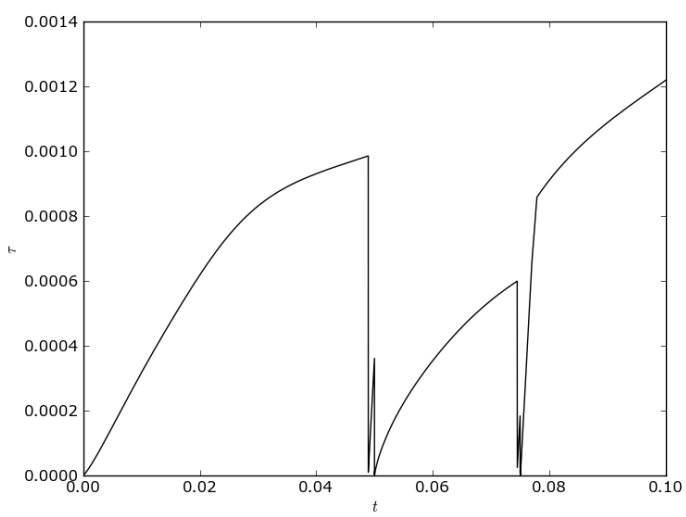

Figure 5. The time-history of the time step: $\delta=0.1, N=569$.

In the region outside the neighbourhood of discontinuities of the coefficients and the right-hand side, the time step is controlled first of all by the term $s_{3}$ (see Fig. 6).

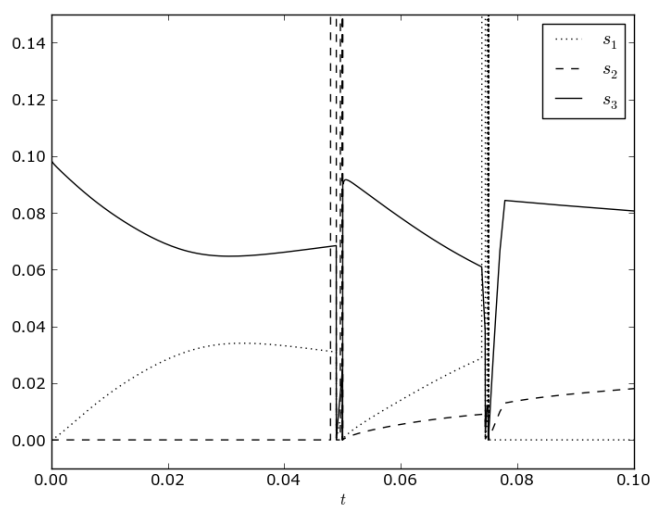

Figure 6. Correcting terms.

A more difficult situation for the numerical solution is connected with inconsistent initial and boundary conditions. Let us consider

$$
u_{0}(x)=1, \quad 0<x<1 .
$$

The selection of the time step for this case is shown in Fig. 7. Up to $t=0.000856$ the calculation is carried out with the minimum time step $\tau_{0}=1 \cdot 10^{-6}$. That is why the total number of time steps is 2183 .

We have presented the results of numerical experiments, which demonstrate the performance of the new strategy of choice of the time step at the hard 


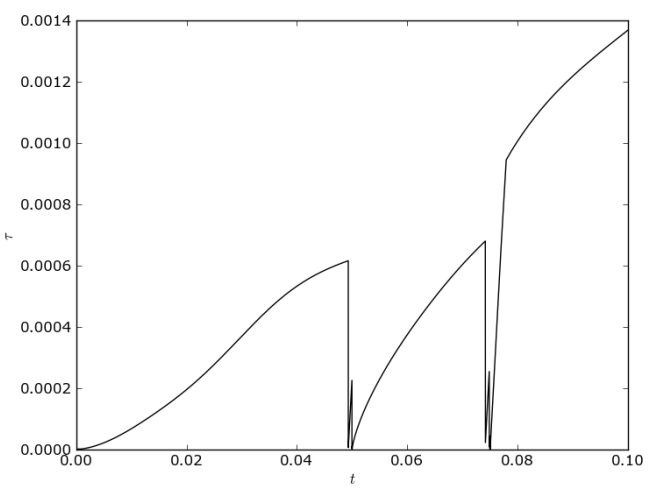

Figure 7. The time-history of the time step: $u_{0}(x)=1$.

conditions, i.e., discontinuity of the coefficients and the right-hand side of the parabolic equation, the presence of the boundary layer at $t=0$. Here we discuss a calculation, which shows the possibility of the algorithm in the case without abrupt changes of the time step.

Let us consider the problem (5.1)-(5.3), where

$$
p(t)=0, \quad f(t)=\cos \left(\frac{4 \pi t}{T}\right) .
$$

Changing the time step at different levels of accuracy $\delta$ is shown in Fig. 8.

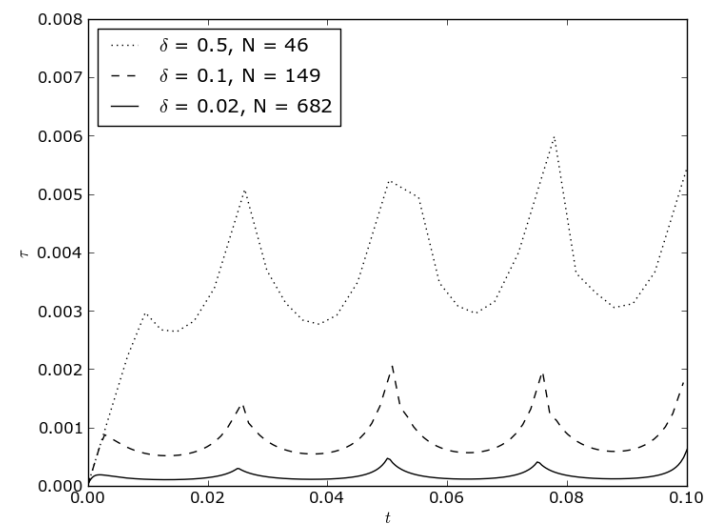

Figure 8. The time-history of the time step for the periodic right-hand side.

Accounting features of the problem rerults in the choice of a smaller step in the high-gradient region of the right-hand side and a fairly rapid transition to a nearly periodic set of time steps. 


\section{Generalizations}

The above-mentioned approach to the adaptive choice of the time step is illustrated by considering the Cauchy problem (2.1), (2.2) and the implicit scheme (2.4), (2.5). Possibilities of using this technique in other conditions are also discussed.

\subsection{The general problem}

Consider more general problems, where instead of (2.1), we study the equation

$$
B(t) \frac{d u}{d t}+C(t) u=g(t), \quad 0<t \leq T
$$

in a finite-dimensional Hilbert space $H$. Assume that in $H$

$$
C(t) \geq 0, \quad B(t)=B^{*}(t) \geq \chi E, \quad \chi>0,
$$

where $E$ is the unit (identity) operator.

To solve numerically the problem (2.2), (6.1), we apply the implicit scheme

$$
B_{n+1} \frac{y_{n+1}-y_{n}}{\tau_{n+1}}+C_{n+1} y_{n+1}=g_{n+1}, \quad n=0,1, \ldots, N-1,
$$

starting from the initial condition (2.5).

The time step in (2.5), (6.2) is estimated using the solutions of the auxiliary problems obtained by means of the explicit scheme. In the forward-backward estimation of the time step, we solve (see (3.2), (3.3)) the following problems:

$$
\begin{gathered}
B_{n} \frac{v_{n+1}-y_{n}}{\tau_{n+1}}+C_{n} y_{n}=g_{n}, \\
B_{n+1} \frac{v_{n+1}-v_{n}}{\tau_{n+1}}+C_{n+1} v_{n+1}=g_{n+1} .
\end{gathered}
$$

Note that for using explicit schemes, we must evaluate $B_{n}^{-1} v$. We assume that the calculation of $B_{n} v, B_{n+1} v$ is not significant in comparison to the implementation of the fully implicit scheme, where it is necessary to calculate $\left(B_{n+1}+\tau_{n+1} C_{n+1}\right)^{-1} v$. Just in this case our technology is appropriate to select the time step without loss of efficiency. The equations (6.3), (6.4) may be written in the form (3.2), (3.3) with

$$
A_{n}=B_{n}^{-1} C_{n}, \quad f_{n}=B_{n}^{-1} g_{n} .
$$

This allows to conduct calculations with the time step determined by the formula (3.10).

\subsection{Higher-order time-stepping methods}

To solve numerically boundary value problems for parabolic equations (2.1), (2.2), in addition to fully implicit scheme (2.4), we can apply the symmetric (Crank-Nicolson) scheme:

$$
\frac{y_{n+1}-y_{n}}{\tau_{n+1}}+A_{n+1} \frac{y_{n+1}+y_{n}}{2}=\frac{f_{n+1}+f_{n}}{2}, \quad n=0,1, \ldots, N-1 .
$$


Its potential benefit is associated primarily with the second-order approximation in time.

To estimate the time step in the scheme $(2.5)$, (6.5), it is necessary to perform this using a scheme of second order, too. Continuing to be in the class of explicit two-level schemes, instead of (3.2), (3.3), we use

$$
\begin{aligned}
& \frac{v_{n+1}-y_{n}}{\tau_{n+1}}+\frac{A_{n+1}+A_{n}}{2} y_{n}-\frac{\tau_{n+1}}{2} A_{n}^{2} y_{n}=\frac{f_{n+1}+f_{n}}{2}-\frac{\tau_{n+1}}{2} A_{n} f_{n} \\
& \frac{v_{n+1}-v_{n}}{\tau_{n+1}}+\frac{A_{n+1}+A_{n}}{2} v_{n+1}+\frac{\tau_{n+1}}{2} A_{n}^{2} v_{n+1}=\frac{f_{n+1}+f_{n}}{2}+\frac{\tau_{n+1}}{2} A_{n} f_{n} .
\end{aligned}
$$

Instead of (6.6), (6.7), we can apply other explicit Runge-Kutta scheme with the second-order accuracy.

The time step is corrected using the explicit calculation formulas that are obtained by constructing the error for the explicit scheme of the same order of accuracy, as the main implicit scheme. In general, it is difficult to obtain explicit formulas for the time step, and the selection strategy can be simplified. We assume that computational costs for the implicit scheme are significantly higher than for the explicit scheme of the same accuracy order. First, for the given prescribed time step $\gamma \tau_{n}$, using explicit schemes, we solve the problem over the intervals $\left[t_{n}, t_{n}+\gamma \tau_{n}\right]$ and $\left[t_{n}+\gamma \tau_{n}, t_{n}\right]$ and obtain $v_{n}$. Next, we estimate the difference between $v_{n}$ and $y_{n}$ and select the step $\tau_{n+1}$. Finally, we find the approximate solution $y_{n+1}$ using the main numerical (implicit) scheme.

\section{Conclusions}

1. A strategy how to control the time step is proposed for solving timedependent problems for parabolic equations. Taking into account the stiffness of a problem, it is based on finding the approximate solution at the new time level using the unconditionally stable implicit approximations in time. To select the time step, auxiliary problems are solved by means of the explicit formulas.

2. To evaluate the time step, we compare the solution at the current time level $t_{n}$ and the approximate solution, obtained by the successive solution of two subproblems over the intervals $\left[t_{n}, t_{n+1}\right]$ and $\left[t_{n+1}, t_{n}\right]$, respectively, using explicit formulas. For the parabolic problems under consideration, the explicit a priori formula is derived for the time step.

3. A similar formula for the time step is obtained by comparing the approximate solutions using explicit formulas when solving the auxiliary problem over the time interval $\left[t_{n}, t_{n+1}\right]$ and the auxiliary problem over two intervals $\left[t_{n}, t_{n+1 / 2}\right]$ and $\left[t_{n+1 / 2}, t_{n+1}\right]$.

4. The robustness of this strategy for selecting the time step is shown for a model parabolic problem. Numerical results demonstrate efficient adaptation to the singular behaviour of the solution at the initial time stage as 
well as to discontinuities in the problem coefficients and in the right-hand side.

\section{References}

[1] L. Angermann and P. Knabner. Numerical Methods for Elliptic and Parabolic Partial Differential Equations. Springer-Verlag, New York, 2003.

[2] U.M. Ascher. Numerical Methods for Evolutionary Differential Equations. Society for Industrial and Applied Mathematics, Philadelphia, 2008.

[3] U.M. Ascher and L.R. Petzold. Computer Methods for Ordinary Differential Equations and Differential-Algebraic Equations. Society for Industrial and Applied Mathematics, Philadelphia, 1998.

[4] W. Bangerth and R. Rannacher. Adaptive Finite Element Methods for Differential Equations. Springer, Basel, 2003.

[5] C.W. Gear. Numerical Initial Value Problems in Ordinary Differential Equations. Prentice Hall, NJ, 1971.

[6] E. Hairer, S.P. Norsett and G. Wanner. Solving Ordinary Differential Equations. I. Nonstiff Problems. Springer-Verlag, Berlin, 1987.

[7] R.J. LeVeque. Finite Difference Methods for Ordinary and Partial Differential Equations. Steady-State and Time-Dependent Problems. Society for Industrial and Applied Mathematics, Philadelphia, 2007.

[8] C.A. Möller. Adaptive Finite Elements in the Discretization of Parabolic Problems. Logos-Verlag, Berlin, 2011.

[9] A.A. Samarskii. The Theory of Difference Schemes. Marcel Dekker, New York, 2001.

[10] A.A. Samarskii and A.V. Gulin. Stability of Difference Schemes. Nauka, Moscow, 1973. in Russian

[11] A.A. Samarskii, P.P. Matus and P.N. Vabishchevich. Difference Schemes with Operator Factors. Kluwer Academic Publ., Dordrecht, 2002.

[12] V. Thomée. Galerkin Finite Element Methods for Parabolic Problems. SpringerVerlag, Berlin, 2010.

[13] R. Verfürth. A Posteriori Error Estimation Techniques for Finite Element Methods. Oxford University Press, Oxford, 2013. 